\title{
Negative regulation of a ribonucleoprotein condensate driven by dilute phase oligomerization
}

\author{
Ian Seim ${ }^{\mathrm{a}, \mathrm{b}, \mathrm{c}}$, Ammon E. Posey ${ }^{\mathrm{d}}$, Wilton T. Snead ${ }^{\mathrm{b}}$, Benjamin M. Stormo ${ }^{\mathrm{b}}$, Daphne Klotsa ${ }^{\mathrm{a}, \mathrm{c}}$, \\ Rohit V. Pappu ${ }^{\mathrm{d}, 1}$, and Amy S. Gladfelter ${ }^{\mathrm{a}, \mathrm{b}, 1}$
}

\begin{abstract}
${ }^{a}$ Curriculum in Bioinformatics and Computational Biology, University of North Carolina at Chapel Hill, Chapel Hill, NC, USA

${ }^{b}$ Department of Biology, University of North Carolina at Chapel Hill, Chapel Hill, NC, USA

'Department of Applied Physical Sciences, University of North Carolina at Chapel Hill, Chapel Hill, NC, USA

${ }^{\mathrm{d}}$ Department of Biomedical Engineering, Center for Science \& Engineering of Living Systems (CSELS), Washington University in St. Louis, St. Louis, MO, USA
\end{abstract}

${ }^{1}$ To whom correspondence may be addressed:pappu@wustl.edu, amyglad@unc.edu 


\begin{abstract}
Ribonucleoprotein bodies are exemplars of membraneless biomolecular condensates that can form via spontaneous or driven phase transitions. The fungal protein Whi3 forms compositionally distinct ribonucleoprotein condensates that are implicated in key processes such as cell-cycle control and cell polarity. Whi3 has a modular architecture that includes a Q-rich intrinsically disordered region and a tandem RNA recognition module. Here, we uncover localized order-todisorder transitions within a 21-residue stretch of the Q-rich region. This region, which can form alpha-helical conformations, is shown to modulate protein density within Whi3-RNA condensates by driving dilute phase oligomerization. Specifically, enhancing helicity within this region enhances oligomerization in the dilute phase. This weakens the associations among disordered Qrich regions thereby diluting the concentration of Whi3 in condensates. The opposite behavior is observed when helicity within the 21-residue stretch of the Q-rich region is abrogated. Thus, dilute phase oligomers, driven by a specific sequence motif, lead to negative regulation of the stoichiometry of protein versus RNA in the dense phase. Our findings stand in contrast to other systems where oligomerization is known to enhance the drive for phase separation. Our results highlight distinctive regulatory effects over phase behavior due to local order-to-disorder transitions within intrinsically disordered regions. This provides a way to leverage molecular scale conformational preferences and coupled intermolecular associations to regulate mesoscale phase behavior and material properties of condensates.
\end{abstract}

\title{
Significance
}

A large sub-class of biomolecular condensates are linked to RNA regulation and known as ribonucleoprotein (RNP) bodies. While extensive work has identified driving forces of biomolecular condensates, relatively little is known about negative regulation of assembly. Here, using a fungal RNP component, Whi3, we show that its intrinsically-disordered, Q-rich region exerts regulatory control over condensate formation through a cryptic helical region that enables the formation of dilute phase oligomers. These oligomers detour Whi3 proteins from condensates, thereby impacting the driving forces for phase separation, the protein-to-RNA ratio in condensates, and the material properties of condensates. Our findings show how nanoscale conformational equilibria can enable control over micron-scale phase equilibria. 
lbody

Intracellular phase transitions are known to drive the formation and dissolution of membraneless biomolecular condensates (1). Prominent condensates include ribonucleoprotein (RNP) bodies such as stress granules $(2-5)$ and processing bodies $(5,6)$ in the cytosol and nuclear bodies such as speckles (7) and nucleoli (8). RNP condensates can form via phase transitions that are influenced by a combination of homotypic and heterotypic protein-protein, protein-RNA, and RNA-RNA interactions (3, 4, 9-13).

The stickers-and-spacers framework provides a way to organize molecular features within protein and RNA molecules and identify their distinctive and collective contributions to the driving forces for phase transitions $(10,14-18)$. In a zeroth order conceptualization, stickers provide specificity and drive percolation transitions via reversible physical crosslinks $(18,19)$. Percolation transitions occur when protein and RNA molecules are topologically connected into system- or condensate-spanning networks. Spacers regulate density transitions, thereby determining whether percolation is aided by phase separation $(18,19)$. Phase transitions driven by multivalent macromolecules that give rise to multicomponent biomolecular condensates are best described as phase separation aided percolation transitions $(15,18,20,21)$.

Recent advances have identified the molecular features within protein (22-26) and RNA sequences $(27,28)$ that drive phase separation-aided percolation. These features include but are not restricted to: the number (valence) of folded stickers $(21,29,30)$ such as RNA recognition motifs (RRMs) $(17,31)$ and protein-protein interaction domains $(1)$; the valence and patterning of short linear motifs (stickers) within intrinsically disordered regions (IDRs) of proteins (7, 22, 24, 26, 32); the physical properties of spacers that are interspersed between stickers (15); and linear interaction motifs, sequence-specific secondary structures, the degree of unfoldedness, and purine versus pyrimidine contents within RNA molecules $(4,9,10,27,28,33)$.

Proteins that drive the formation of RNP condensates via phase separation aided percolation often have modular architectures featuring structured oligomerization domains (SODs), IDRs, and RRMs (3). There are several examples of proteins that contain both IDRs and SODs. Prominent among these are the dimerization domain of the Nucleocapsid protein in SARSCoV-2 (34, 35), the NTF2L dimerization domain of G3BP1/2 (2), a scaffold for stress granule formation, the oligomerization domain within coilin that drives Cajal Body formation (36), and the PB1 domain of auxin response factors (37), which gives rise to condensates that repress transcription during stress in plants. Oligomerization via SODs tethered to IDRs has also been shown to drive phase transitions in engineered systems (38-40). Synthetic and naturally occurring SODs are thought to act as sinks that lower the entropic penalty associated with phase transitions (39). In this view, SODs enable the increased multivalence and local concentration of stickers, which in turn drives phase transitions.

It is well known that many IDRs undergo coupled binding and folding reactions either through heterotypic interactions with cognate binding partners or via homotypic interactions that lead to dimers and higher-order oligomers (41-45). An archetypal example of dimerization induced folding of an IDR is the leucine zipper motif, which has a weak intrinsic preference for alphahelical conformations and forms coiled-coil alpha-helices as dimers $(45,46)$ or higher-order oligomers (47). Residues that define the interface among helices contribute to the oligomerization status of these leucine zipper systems. Overall, the stability of coiled coils is thermodynamically linked to the intrinsic preference for forming alpha-helices as monomers. 
How might sequence-specific preferences for alpha-helical conformations within IDRs and their oligomerization impact the phase behavior of RNA-binding proteins? Recent studies have identified the propensity to form alpha-helical structures within the C-terminal IDR of TDP-43 (48). This has been shown to be a key determinant of the driving forces for phase separation (48). The findings for TDP-43 are reminiscent of findings regarding the effects of oligomerization driven by SODs. The general consensus is that oligomerization via SODs enhances the driving forces for phase separation. Here we uncover a surprising negative regulation of phase separation associated with local ordering within an IDR that drives oligomerization. Our work highlights the distinctive, context-dependent contributions that different types of IDRs and oligomerization make to phase separation.

Our interest is in the phase behavior of the fungal protein Whi3, which forms RNP condensates with different RNA molecules $(9,49)$. The simplest system has two macromolecular components namely, the Whi3 protein and an RNA molecule such as the cyclin mRNA, CLN3. The Whi3 protein encompasses a C-terminal RRM, and a long adjacent IDR that includes a glutamine-rich region (QRR) (Fig. 1A). Sequence analysis shows that the QRR encompasses a 21residue motif, which we designate as $\mathrm{CC}$, that has many of the features associated with alphahelical coiled-coil forming domains (50). This opens the door to the possibility that the phase behavior of Whi3 is regulated by this putative helix- and coiled-coil-forming domain. Our work focuses on an interrogation of the effect of the $\mathrm{CC}$ motif on Whi3 phase behavior.

We characterized the intrinsic helicity of the $\mathrm{CC}$ motif, in isolation, and assessed its impact on the phase behavior of Whi3 through comparative studies of conformational and phase equilibria of mutants that diminish $\left(\mathrm{CC}^{-}\right)$or enhance $\left(\mathrm{CC}^{+}\right)$helicity. Surprisingly, we find that while enhancing intrinsic helicity enhances oligomerization in the dilute phase, it weakens the driving forces for phase separation and lowers the density of Whi3 in the RNP condensates. The converse is true when helicity is diminished. Further spectroscopic investigations help establish the importance of disorder through the $\mathrm{CC}$ region for driving the intermolecular interactions that are important for phase separation. Through coarse-grained simulations, we show how oligomerization and clustering within the dilute phase modulates phase behavior in agreement with experimental observations. Dilute phase oligomers act as off-pathway sinks for Whi3 proteins, whereas disorder within the QRR promotes phase separation through a combination of homotypic and heterotypic interactions. Our studies help identify a distinctive role for local order-to-disorder transitions and highlight the existence of oppositional linkage among conformational equilibria, oligomerization, and phase equilibria.

\section{Results}

The QRR of Whi3 encompasses a region with a weak intrinsic preference for alphahelical conformations. The Whi3 protein encompasses a distinctive, 108-residue QRR (Fig. 1A). Although this region is predicted to be disordered, sequence analysis revealed the presence of a 21-residue stretch, designated as $\mathrm{CC}$, that has the characteristic features of alpha-helical coiledcoil domains $(50,51)$. These features include the absence of Pro and the presence of Leu at every seventh position (Fig. 1A). The putative CC motif appears to be an example of alpha-helical molecular recognition elements / features ( $\alpha$-MoREs or $\alpha$-MoRFs) that are found in many IDRs $(52,53)$. Atomistic simulations based on the ABSINTH implicit solvation model and forcefield paradigm (54) have proven to be useful for detecting $\alpha$-MoREs / $\alpha$-MoRFs in IDRs $(55,56)$. 
We performed atomistic simulations on the 21-residue sequence CC peptide. The sequence of this peptide is shown in Fig. 1A. A series of independent simulations were performed at different temperatures. For the $\mathrm{CC}$ peptide, the median helicity is low with low variance at high and low temperatures. However, the median helicity peaks for an intermediate temperature range (Fig. 1B). We quantified the sequence-specificity of the alpha-helicity of the $\mathrm{CC}$ peptide by introducing point mutations that are likely to diminish / enhance helicity. The two designed peptides are designated as $\mathrm{CC}^{-}$and $\mathrm{CC}^{+}$, respectively. The three Leu residues in the original $\mathrm{CC}$ peptide were replaced with Pro to generate $\mathrm{CC}^{-}$, a sequence variant designed to show diminished alpha helicity. Conversely, we replaced the fourth residue of each heptad repeat in the $\mathrm{CC}$ peptide with a Leu to generate $\mathrm{CC}^{+}$. As expected, the simulations show significantly diminished helicity for $\mathrm{CC}^{-}$and enhanced helicity for $\mathrm{CC}^{+}$. These results are illustrated in Fig. 1C and Fig. 1D, respectively. As a calibration, the CC peptide has a median helicity of $\sim 30 \%$ at $\sim 320 \mathrm{~K}$. At a similar temperature, the median helicities of $\mathrm{CC}^{-}$and $\mathrm{CC}^{+}$are $15 \%$ and $60 \%$, respectively.

To test predictions from the simulations, we performed ultraviolet circular dichroism (UVCD) measurements for the three peptides (see methods for details). These spectra were collected at different temperatures and the data are shown in Figs. 1E-G. Across the temperature range of interest, the spectra for the CC peptide show two minima at $208 \mathrm{~nm}$ and $222 \mathrm{~nm}$, respectively. The molar ellipticity is lower for the minimum at $208 \mathrm{~nm}$. This is consistent with a heterogeneous ensemble with significant alpha-helical character $(55,56)$, although the helix is not a persistent conformation. In contrast, and in accord with our predictions, each of the spectra for $\mathrm{CC}^{-}$show only a single minimum at $\sim 205 \mathrm{~nm}$. There is a positive peak in the $215-218 \mathrm{~nm}$ range at low temperatures and this is concordant with the presence of tertiary amides i.e., Pro residues within the sequence (57). Overall, the spectra for $\mathrm{CC}^{-}$are consistent with this peptide being disordered.

Finally, the spectra for the $\mathrm{CC}^{+}$peptide demonstrate the characteristic minima at $208 \mathrm{~nm}$ and $222 \mathrm{~nm}$. In addition, at temperatures below $338 \mathrm{~K}$, the molar ellipticity at $222 \mathrm{~nm}$ is lower than the molar ellipticity at $208 \mathrm{~nm}$. The molar ellipticities between $200 \mathrm{~nm}$ and $240 \mathrm{~nm}$ become less negative with increasing temperature and the presence of an isodichroic point at $200 \mathrm{~nm}$ is suggestive of a canonical helix-to-coil transition (58) for $\mathrm{CC}^{+}$as temperature increases. By fitting the spectra for each peptide to a two-state model, we estimate the melting temperatures for helixto-coil transitions to be $333 \mathrm{~K}$ and $243 \mathrm{~K}$ for $\mathrm{CC}^{+}$and CC, respectively (Fig. S1, SI Appendix). This implies that $\mathrm{CC}$ populates a heterogenous conformational ensemble at physiological temperatures, whereas $\mathrm{CC}^{+}$is considerably more alpha-helical under these conditions. In contrast, $\mathrm{CC}^{-}$has negligible preference for alpha-helical conformations (Fig. 1F). 

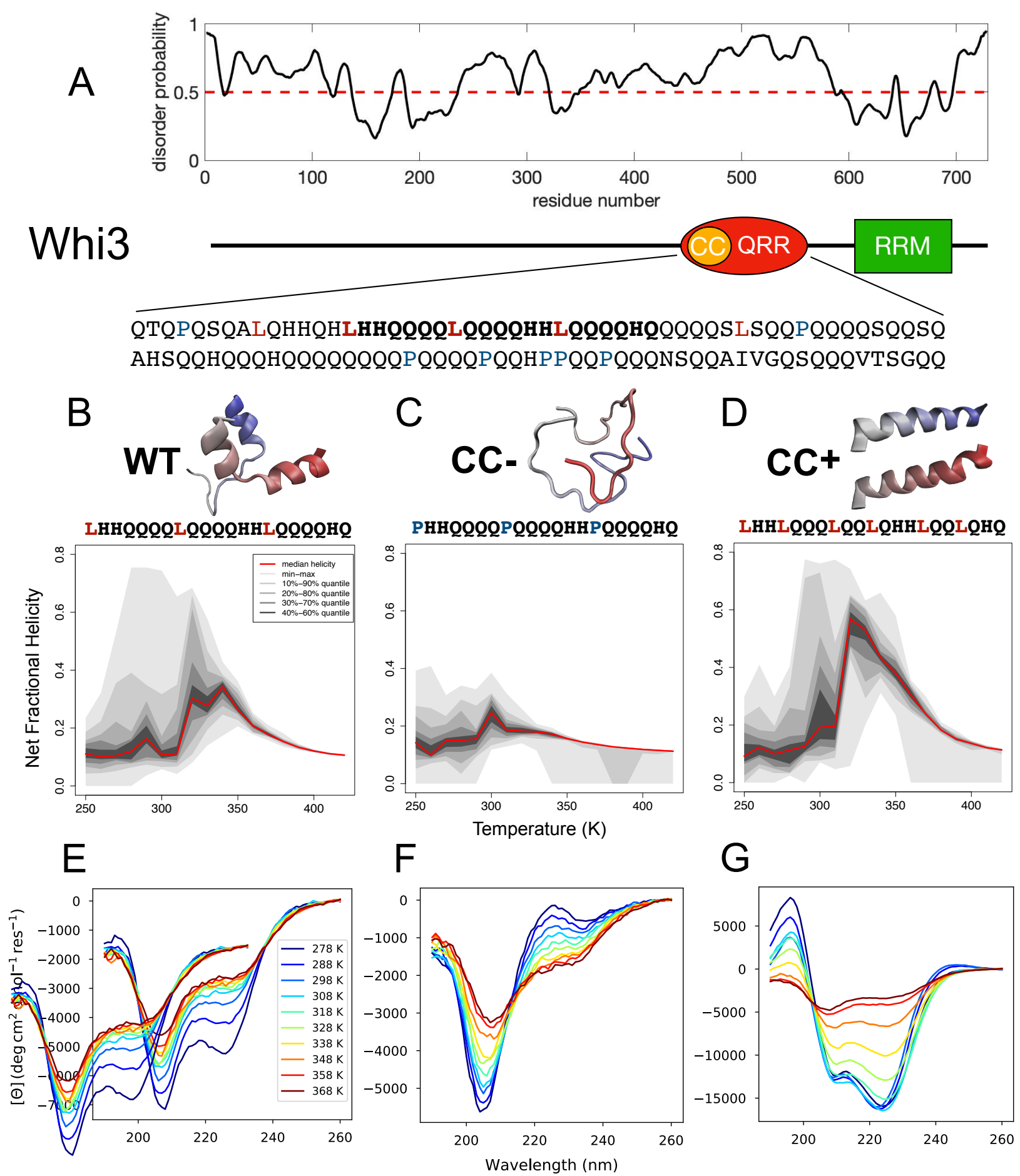

Figure 1: The QRR of Whi3 encompasses a putative CC motif. (A) Whi3 is predicted to be highly disordered (PrDOS). The sequence includes a 108-residue QRR. The bold-faced region corresponds to the $\mathrm{CC}$ motif. Leu residues are colored in red. Note the exclusion of Pro (in blue) from the $\mathrm{CC}$ motif. (B-D) Each panel is annotated by representative conformations extracted from simulations of the $\mathrm{CC}, \mathrm{CC}^{-}$, and $\mathrm{CC}^{+}$peptides. The fractional helicity profiles within each panel show results from 20 independent simulations performed at each of the simulation temperatures. The results are shown with quantiles and the red curves show the computed median helicities. (E- 
G) UV-CD spectra, measured at a series of temperatures, for the $\mathrm{CC}, \mathrm{CC}^{-}$, and $\mathrm{CC}^{+}$peptides, respectively.

The phase behavior of Whi3 is modulated by the CC motif. To assess the impact of the CC motif on the phase behavior of Whi3, we studied the co-phase separation of Whi3 in the presence of the cyclin RNA CLN3 (9) and compared this to the measured phase behavior obtained for three distinct variants. We purified full-length wild-type Whi3 (WT), and three variants namely, Whi3 $\left(\mathrm{CC} \rightarrow \mathrm{CC}^{-}\right)$, Whi3 $\left(\mathrm{CC} \rightarrow \mathrm{CC}^{+}\right)$, and Whi3 $(\triangle \mathrm{CC})$. In the Whi3 $(\triangle \mathrm{CC})$ construct, we deleted the 21 residues of the $\mathrm{CC}$ motif within Whi3. In each of our measurements, the Whi3 proteins and CLN3 RNA molecules have fluorescent dye labels, and these are used to assess dense phase concentrations. In the images, Whi3 (green) and CLN3 (magenta) channels are contrasted such that fluorescence is proportional to concentration, providing a visual readout of dense phase stoichiometry.

Changes to the $\mathrm{CC}$ sequence resulted in significant differences in the dense phase stoichiometry (Fig. 2A). We quantified the concentrations of Whi3 and CLN3 in the dense phase by measuring fluorescence intensities and comparing to calibration curves (Fig. S2, SI Appendix). Condensates across all systems contain roughly the same dense phase concentration of CLN3. However, we observe significant variation in the concentrations of Whi3 within dense phases. Condensates formed by Whi3 $\left(\mathrm{CC} \rightarrow \mathrm{CC}^{-}\right)$and Whi3 $(\Delta \mathrm{CC})$ have twice the dense phase protein concentration when compared to WT. In contrast, the protein concentration in condensates formed by Whi3 $\left(\mathrm{CC} \rightarrow \mathrm{CC}^{+}\right)$is a fifth of that of WT (Fig. 2B). This indicates that increased helicity in the $\mathrm{CC}$ motif leads to lower concentrations of Whi3 in the dense phase. Conversely, a disordered $\mathrm{CC}$ motif or deletion of the CC motif increases the dense phase concentration of Whi3.

Next, we assessed whether the impact on changes to protein concentrations in condensates arises from homotypic versus heterotypic interactions. Across the four constructs, we observe changes in the dense phase concentration of Whi3, while the concentration of CLN3 within condensates remains constant (Fig. 2B). Accordingly, we hypothesized that the effects of the mutations within the CC motif should be evident even in the absence of CLN3. To test this hypothesis, we performed phase separation assays in the absence of CLN3. In line with results from the Whi3-CLN3 system, we observe robust phase separation for Whi3 $\left(\mathrm{CC} \rightarrow \mathrm{CC}^{-}\right)$, intermediate levels of phase separation for WT, and weak phase separation for Whi3 $\left(\mathrm{CC} \rightarrow \mathrm{CC}^{+}\right)$ (Fig. 2C, D). Moreover, the dense phase concentration increased for Whi3 $\left(\mathrm{CC} \rightarrow \mathrm{CC}^{-}\right)$and decreased for Whi3 $\left(\mathrm{CC} \rightarrow \mathrm{CC}^{+}\right)$, consistent with results from the Whi3-CLN3 system. Clearly, the $\mathrm{CC}$ motif contributes to the regulation of Whi3 phase behavior, and it does so through homotypic interactions. 

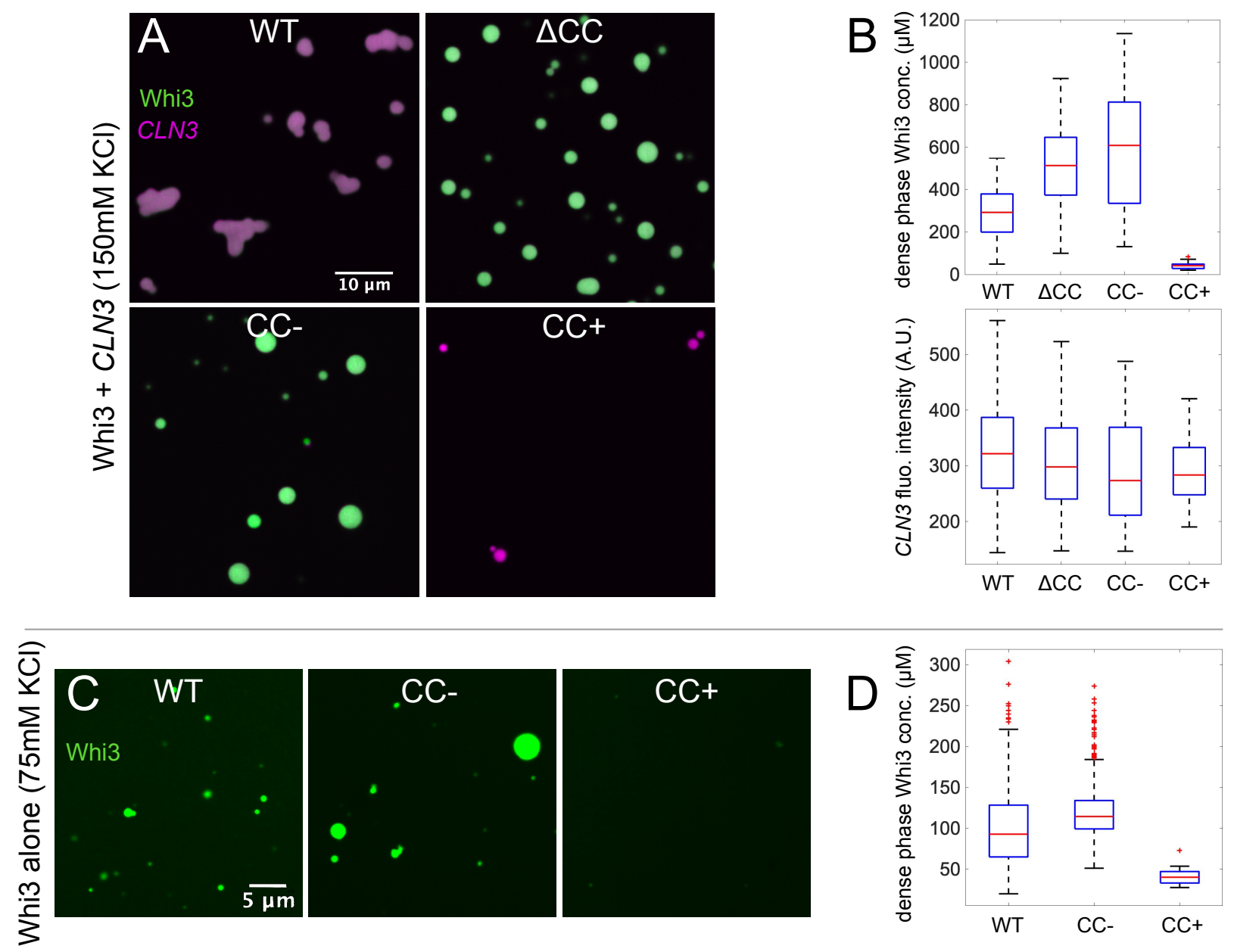

Figure 2: CC mutations control Whi3-CLN3 phase behavior. (A) Representative images of each construct at five hours after mixing $500 \mathrm{nM}$ Whi3 with $5 \mathrm{nM} C L N 3$. Whi3 (green) and CLN3 (magenta) channels are merged and contrasted such that brightness is proportional to concentrations. (B) Dense phase Whi3 concentration and $C L N 3$ fluorescence intensity calculated for each condensate in experiments corresponding to panel (A). (C) Whi3-only phase separation recorded 4 hours after mixing. All images are contrasted such that fluorescence intensity is proportional to protein concentration. (D) Dense phase Whi3 concentrations at a bulk concentration of $17 \mu \mathrm{M}$.

The CC motif enables sequestration of the Whi3 protein into dilute phase oligomers. To arrive at a mechanistic understanding for the observed effects of the $\mathrm{CC}$ motif on Whi3 phase behavior, we modeled the CC and QRR domains of Whi3 using the LASSI stickers and spacers model (14) (Fig. 3A). For simplicity, we focused on a minimal system comprising the CC-QRR fragment. We assume that $\mathrm{CC}$ is a dimerization motif that can access both dimerization competent and dimerization incompetent states. In this model, the $\mathrm{CC}$ dimerizes with other $\mathrm{CC}$ domains via anisotropic interactions. This represents interactions between folded molecules. In addition, the CC motif functions like the rest of the QRR and engages in weak, isotropic interactions representing the unfolded state. To represent the effects of mutations on the helicity and dimerization of the CC motif, we implement a trade-off between the energies associated with isotropic versus anisotropic interactions involving the beads that mimic the CC motif in the model 
(Fig. 3A). The right-most system in Fig. 3 represents the extreme case that is most like Whi3 $\left(\mathrm{CC} \rightarrow \mathrm{CC}^{+}\right)$, with a perfectly stable helix, and strong anisotropic binding. The central system corresponds to the opposite limit, mimicking Whi3 $\left(\mathrm{CC} \rightarrow \mathrm{CC}^{-}\right)$, with a completely unfolded $\mathrm{CC}$ motif that interacts purely via isotropic interactions. The WT system has a CC bead that interacts via a combination of weaker anisotropic and isotropic interaction energies.

For each system, we performed a series of LASSI simulations across a range of concentrations and temperatures. We compute coexistence curves i.e., binodals, using the approach prescribed by Choi et al. (14). The computed phase boundaries show that changes to the interactions mediated by the $\mathrm{CC}$ bead are sufficient to alter the driving forces for phase separation (Fig. 3B). We observe a suppression of phase separation when the isotropic interactions are weakened in favor of strengthening the anisotropic interactions. The converse is true when the isotropic interactions are strengthened over anisotropic interactions. This behavior is consistent with results from measurements that were performed in the absence of CLN3 (Fig. 2D).

For each of the simulations, we quantified the distributions of cluster sizes in the coexisting dilute phases. Enhancement of CC dimerization contributes to an increase in the sizes of dilute phase oligomers (Fig. 3C). Although the $\mathrm{CC}$ beads can only form dimers, they are connected to QRRs within the chain molecules. Accordingly, increasing the strength of anisotropic interactions of CC interactions increases the local concentration of appended QRRs, leading to enhanced interactions among QRRs. This leads to large, dilute phase oligomers that act as sinks for Whi3 molecules. These sinks weaken the driving forces for Whi3 phase separation and may be viewed as unproductive species as far as Whi3 phase separation is concerned.

To test predictions from the LASSI simulations, we measured oligomer sizes for WT, Whi3 $\left(\mathrm{CC} \rightarrow \mathrm{CC}^{-}\right)$, and Whi3 $\left(\mathrm{CC} \rightarrow \mathrm{CC}^{+}\right)$at low concentrations that correspond to the one-phase regime (Fig. 3D). Using the fluorescence of single Whi3 molecules as a calibration, we recorded the distribution of oligomer sizes for each protein. Our predictions from LASSI-based simulations are supported by the observation that, despite their greatly reduced phase separation ability, Whi3 $\left(\mathrm{CC} \rightarrow \mathrm{CC}^{+}\right)$constructs form large oligomers in dilute settings.

Taken together, our results indicate that the $\mathrm{CC}$ motif functions as a regulatory module that can sequester protein into the dilute phase, thereby impacting the driving forces for phase separation and altering the dense phase Whi3 concentration. By changing the helicity of CC, the balance of protein between the two phases is shifted. This is observed in the different Whi3-CLN3 systems, indicating that the protein density in Whi3-RNA condensates is impacted, in part, by homotypic associations among Whi3 molecules. 
A
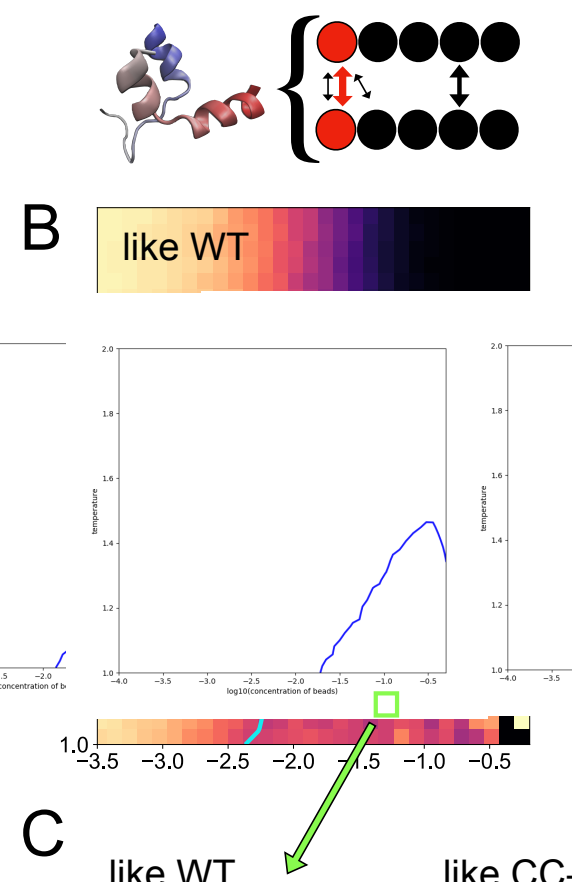

like WT like CC-

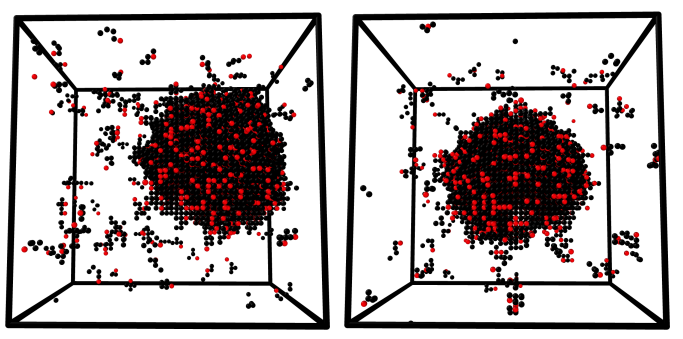

D

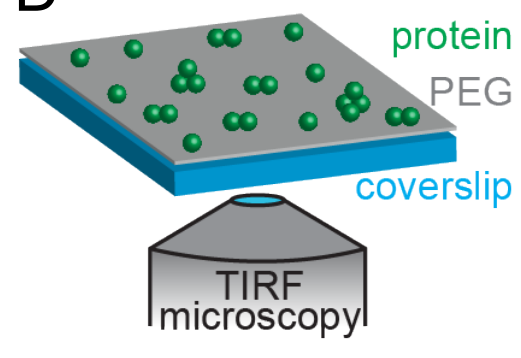

$\begin{array}{ll}=\text { coiled-coil } & \mathfrak{t}=\text { isotropic } \\ =\text { QRR } & \mathfrak{\imath}=\text { anisotropic }\end{array}$
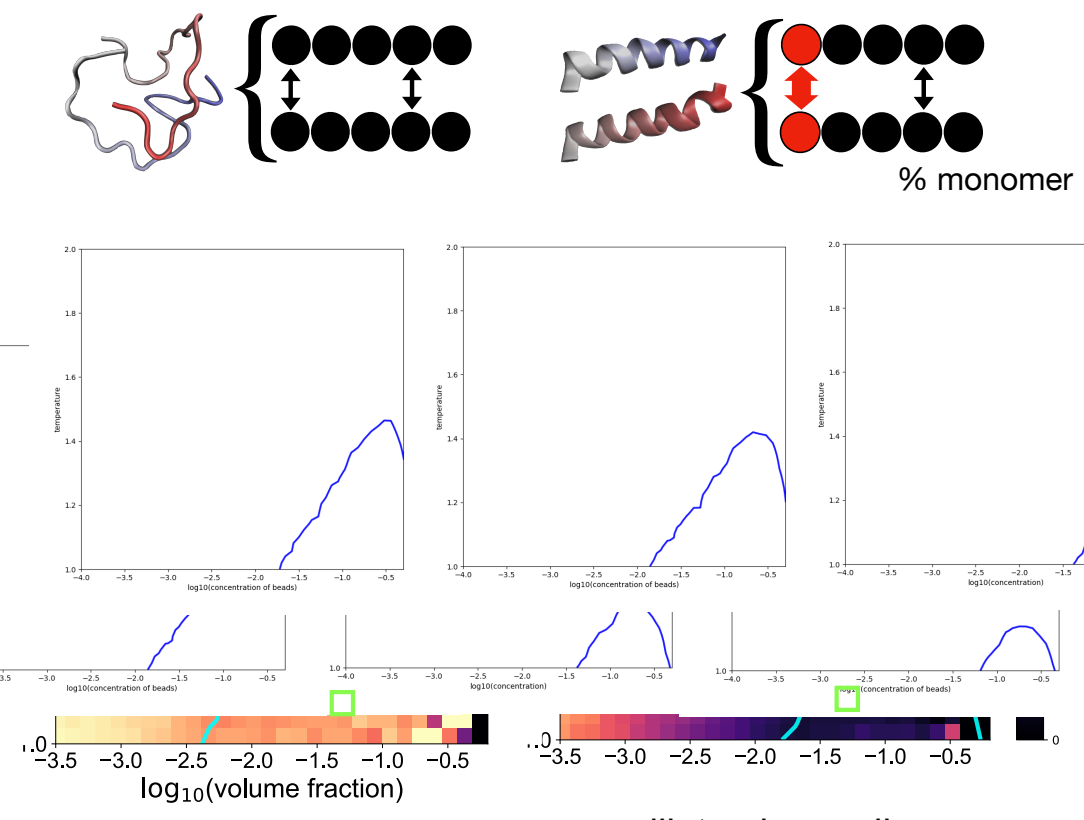

dilute phase oligomers
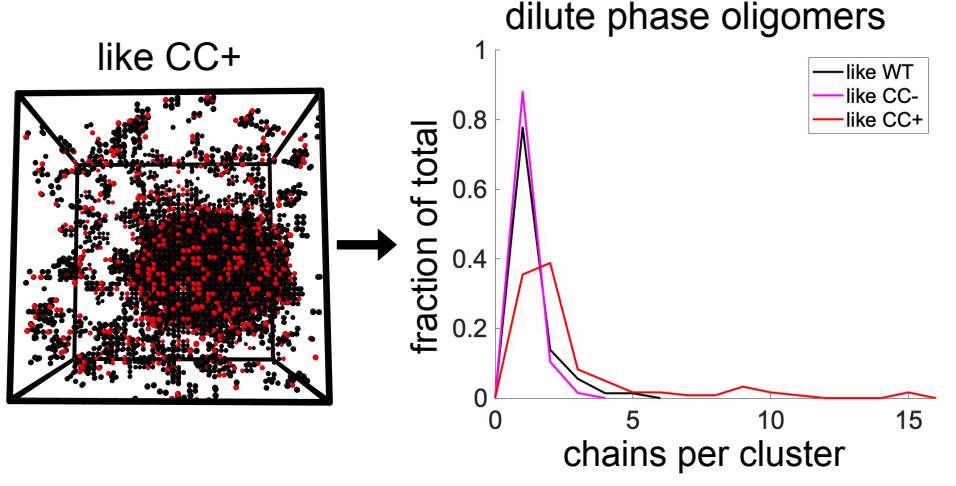
Evidence for loss of helicity driving phase separation. Our data to this point suggest that stable helix formation within the $\mathrm{CC}$ motif should sequester Whi3 molecules in dilute phase oligomers that form via strong $\mathrm{CC}$-mediated interactions and are further stabilized by weaker interactions among QRRs. In contrast, a lack of helicity within the CC motif drives phase separation, which leads to higher protein concentrations in dense phases characterized by nonstoichiometric assemblies. Inspired by work of Urry and coworkers $(59,60)$, as well as that of Duysens (61), we tested the hypothesis that loss of helicity through the CC motif drives phase separation, which has been referred to as coacervation in the early literature. For this, we turned to UV-CD measurements and collected spectra as a function of peptide / protein concentration. The constructs studied include the three peptides $\mathrm{CC}, \mathrm{CC}^{-}$, and $\mathrm{CC}^{+}$as well as the full-length Whi3 protein. Systems that undergo phase separation are known to show a strong distortion of the CD spectra that is accompanied by a red shift, a flattening of the spectra, and the appearance of a new minimum at $\sim 230 \mathrm{~nm}(61)$. Urry and coworkers $(59,60)$ noted that these features of CD spectra are related to the increased sizes of particles in solution, protein concentration, and composition. Accordingly, we used UV-CD spectroscopy to answer the following questions: Do we observe the predicted distortions viz., red-shifting, flattening of spectra, and the appearance of an auxiliary minimum near $230 \mathrm{~nm}$ at high peptide / protein concentrations? And are these changes, which point to the onset of phase separation, accompanied by a loss of helicity?

The results of our CD titrations are shown in Fig. 4. Data for the CC peptide show the red shift, which is accompanied by a flattening of the spectra, and the appearance of a new minimum near $230 \mathrm{~nm}$. These changes are manifest above a threshold concentration (Fig. 4A) that serves as a precursor of phase separation. The onset of the distortion of CD spectra is also observed for the $\mathrm{CC}^{-}$and $\mathrm{CC}^{+}$peptides (Fig. 4B, C). The $\mathrm{CD}$ spectra for the $\mathrm{CC}^{-}$peptide, which has negligible helicity, show monotonic changes as a function of peptide concentration with the appearance of distorted spectra being manifest at the lowest concentrations of the three peptides studied here (Fig. 4B). For $\mathrm{CC}^{+}$, the distortion follows the loss of helicity, which is realized as the concentration goes above a threshold (Fig. 4C). We can compare the extent to which each of the peptides drive coacervation through unfolded conformations by comparing the spectra collected at similar peptide concentrations (Fig. 4D). This comparison reveals that the extent of distortion, red shift, and flattening, i.e., precursor signatures of coacervation, are most pronounced for $\mathrm{CC}^{-}$followed by $\mathrm{CC}$, and then $\mathrm{CC}^{+}$. This result is consistent with expectations that increased helicity detours peptides away from forming coacervates by sequestering them into dilute phase oligomers. In contrast, the interactions among unfolded peptides appear to drive coacervation.

Next, we asked if the observations reported for the peptides could be recapitulated for the full length Whi3 protein. Accordingly, we measured concentration dependent CD spectra for the full-length Whi3 protein (Fig. 4E) at three different concentrations. At low concentrations, the contribution of the folded RRM is apparent in the CD spectrum. However, as concentrations increase, we observe the expected flattening, red shift, and appearance of a new minimum at $\sim 230$ $\mathrm{nm}$. We note that the assemblies observed in these CD experiments are precursors of coacervates, since coacervates themselves scatter too much light and fall out of solution. Our data suggest that unfolding is a pre-requisite for the increased multivalence of stickers that is required to drive Whi3 phase separation. These data are in accord with the findings from the LASSI-based simulations, wherein folded states suppress phase separation via stronger, anisotropic interactions. 

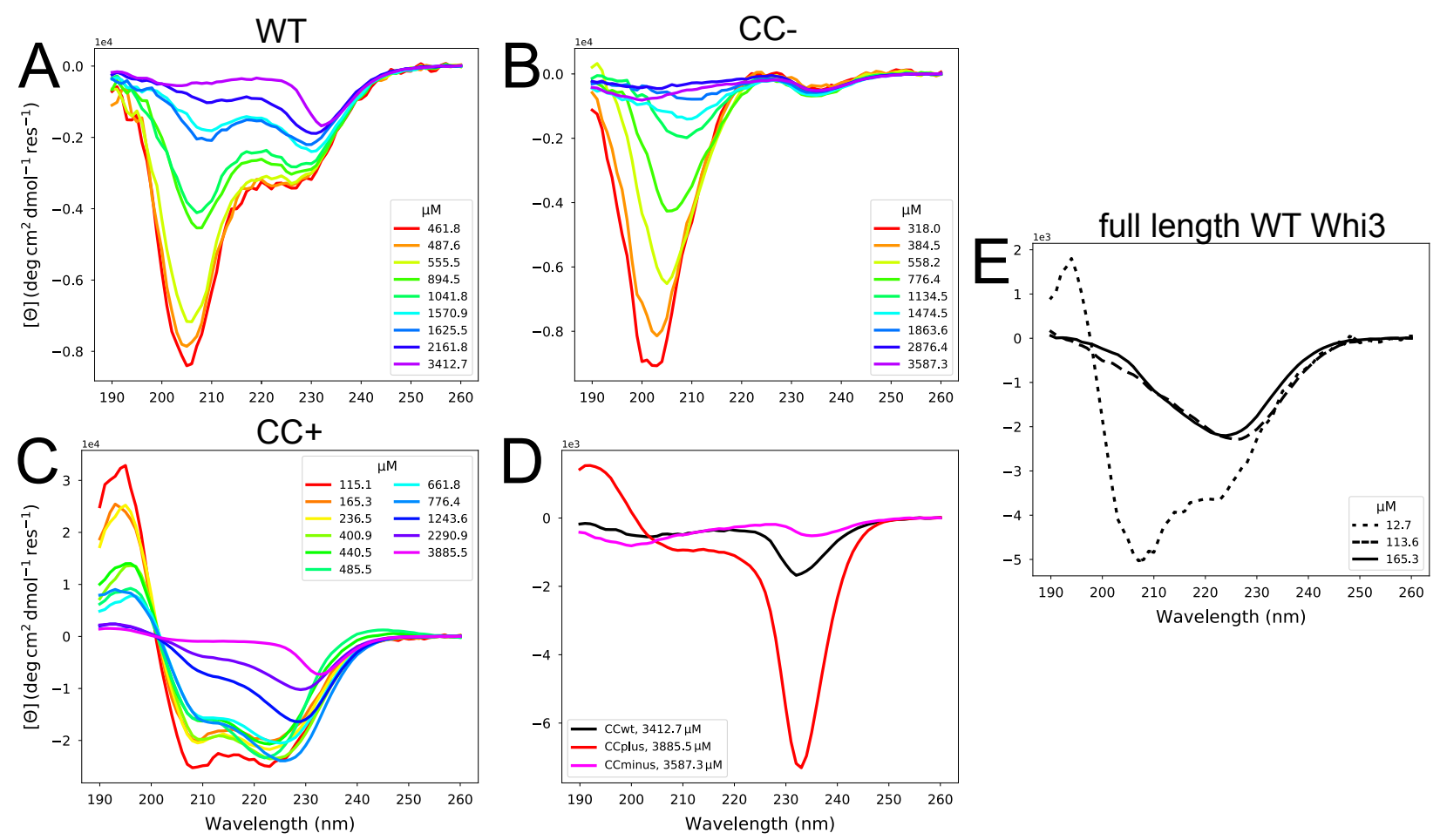

Figure 4: Concentration-dependent $C D$ spectra reveal differences in conformational and assembly states across the different $\mathrm{CC}$ peptides and full-length WT Whi3. (A) CD spectra for the WT CC peptide. (B) CD spectra for the $\mathrm{CC}^{-}$peptide. (C) CD spectra for the $\mathrm{CC}^{+}$peptide. (D) CD spectra at the highest concentrations measured for each peptide. (E) CD spectra are shown for full-length WT Whi3 at three concentrations.

Dense phase concentrations correlate with material states of condensates. In addition to changes in dense phase stoichiometry and dilute phase oligomer distributions, droplet morphology in the Whi3-CLN3 systems varied with time and across constructs, with each system showing several dynamical regimes. An hour after mixing, the condensates are small and highly mobile. At intermediate stages of coarsening, droplets collide and partially coalesce (Fig. 5A). WT condensates have irregular shapes at the five-hour mark and later, while Whi3 ( $\left.\mathrm{CC} \rightarrow \mathrm{CC}^{-}\right)$ condensates are more spherical at all times and completely round up into spheres within four hours.

We quantified condensate geometry at each time point using a custom image analysis pipeline (see methods). The Whi3 $\left(\mathrm{CC} \rightarrow \mathrm{CC}^{-}\right)$and Whi3 $(\Delta \mathrm{CC})$ systems have larger average condensate areas at early times and higher circularity at all times relative to WT. This is consistent with the observation that Whi3 $\left(\mathrm{CC} \rightarrow \mathrm{CC}^{-}\right)$condensates coalesce more rapidly (Fig. 5B). On the other hand, Whi3 $\left(\mathrm{CC} \rightarrow \mathrm{CC}^{+}\right)$condensates demonstrate distinct coarsening behavior, with very small average sizes that plateau at two hours and high circularity values at all times (Fig. 5B). This is because droplets formed by Whi3 $\left(\mathrm{CC} \rightarrow \mathrm{CC}^{+}\right)$collide at early stages of phase separation (1-2 hrs), but instead of coalescing they stick to one another without relaxing, (Fig. S3 in SI Appendix) or bounce off each other. Taken together, the Whi3-CLN3 in vitro data demonstrate a correlation between the dense phase stoichiometry and the material state of condensates. Specifically, higher Whi3-CLN3 concentration ratios within condensates leads to more round droplets that coalesce more quickly. We also observed that higher bulk concentrations of Whi3 with $5 \mathrm{nM} C L N 3$ led to more Whi3 in dense phase droplets (Fig. S4 A-C), as expected for ternary systems (Fig. S5A). 
Droplets with more Whi3 also rounded up and coarsened more quickly (Fig. S4 A-C). These data further demonstrate a correlation between dense phase stoichiometry and material states.

Previous work showed that Whi3-CLN3 condensates age, resulting in slowed dynamics and fibril-like structures within droplets at long times (33). To explore the dynamics of maturation, we modeled the dynamic evolution of the different Whi3 variants systems using the ternary CahnHilliard-Cook system of equations (62), coupled to a gelation process:

$$
\begin{gathered}
\frac{\partial \phi_{i}}{\partial t}=\nabla \cdot\left(M_{\phi_{i}}(c) \nabla\left(\frac{\partial F_{F H}}{\partial \phi_{i}}-\epsilon^{2} \nabla^{2} \phi_{i}\right)\right)+\sigma \cdot \zeta \\
\frac{d c}{d t}=M_{c}\left(g\left(\phi_{p}, \phi_{r}\right) c-c^{2}\right), \quad M_{\phi_{i}}(c)=\exp \left(-c / c_{\phi_{i}}\right)
\end{gathered}
$$

Here, $F_{F H}$ is the ternary Flory-Huggins free energy, and $g\left(\phi_{p}, \phi_{r}\right)$ defines the parabolic sol-gel contours shown in Fig. 5C (derivations in SI Appendix). This model provides a link between dense phase stoichiometry and aging by locating dense phase concentrations vis-à-vis the sol-gel line. At equilibrium, condensates formed by associative polymers with sticker-and-spacer architectures are defined by condensate spanning networks. However, the gap between the sol-gel line and the dense phase arm of the phase boundary (63) can lead to gelation without phase separation $(64,65)$, which corresponds to dynamically arrested phase separation. If the gap between the sol-gel line and the dense phase arm of the phase boundary is large (63), then gelation without phase separation is readily realized via dynamical arrest of phase separation $(16,63)$. This results in rapid aging, irregular morphologies, and ultraslow relaxation to spherical morphologies $(10,66)$. Thus, the material state and resulting dynamics of condensates can be tuned by altering the location of the dense phase arm and hence the gap between the sol-gel line and the dense phase arm (63).

We can tune dense phase stoichiometries by changing the magnitude of Whi3 homotypic interactions in the Flory-Huggins free energy (Fig. S5 B, C in SI Appendix). The Flory-Huggins free energy represents averaged interactions among components, so the Whi3 homotypic interaction in this context encompasses contributions from both the CC and QRR. The strength of the homotypic interactions that drives phase separation is implicitly linked to the degree of unfoldeness of the $\mathrm{CC}$ motif and other regions of Whi3. Accordingly, in the FH model, when the Whi3 homotypic interaction strength is low, the dense phase arm is furthest beyond the sol-gel line, and droplet dynamics are arrested quickly, as in the Whi3 $\left(\mathrm{CC} \rightarrow \mathrm{CC}^{+}\right)$system (Fig. 5 C, D). Alternatively, when the Whi3 homotypic interactions strength is high, the gap between the dense phase concentration and sol-gel line is narrowed, and the dynamics are minimally affected (Fig. 5 C, D). We observed similar effects when altering Whi3 bulk concentration in the model, where high Whi3 bulk concentrations lead to high dense phase Whi3 concentrations and coarsening over longer times (Fig. S4 D, E, SI Appendix). These results are a natural consequence of a ternary phase boundary intersecting the sol-gel line. They represent the influence of stoichiometry on gelation.

Since our model does not include Brownian motion of droplets, coalescence is not an important factor in coarsening. Instead, coarsening is driven by Ostwald ripening (67), which is hindered as gelation arrests phase separation. Thus, droplet circularity is not affected by gelation in this model, but droplet sizes are, such that smaller sizes indicate increased gelation that arrests phase separation due to the formation of percolated droplets. We performed simulations at different homotypic interactions strengths and bulk concentrations without the gelation term, an 
approximation that corresponds to phase separation without gelation. In this scenario, we do not find significant differences in average droplet sizes (Fig. S6, SI Appendix). Results from this model are consistent with the experimental observation that a higher dense phase Whi3-CLN3 concentration ratio correlates with a more liquid-like state and suggests that the links between dense phase stoichiometries and viscoelastic properties include parabolic sol-gel contours and the sequence-specific gaps between sol-gel lines and dense phase arms of phase boundaries (63).
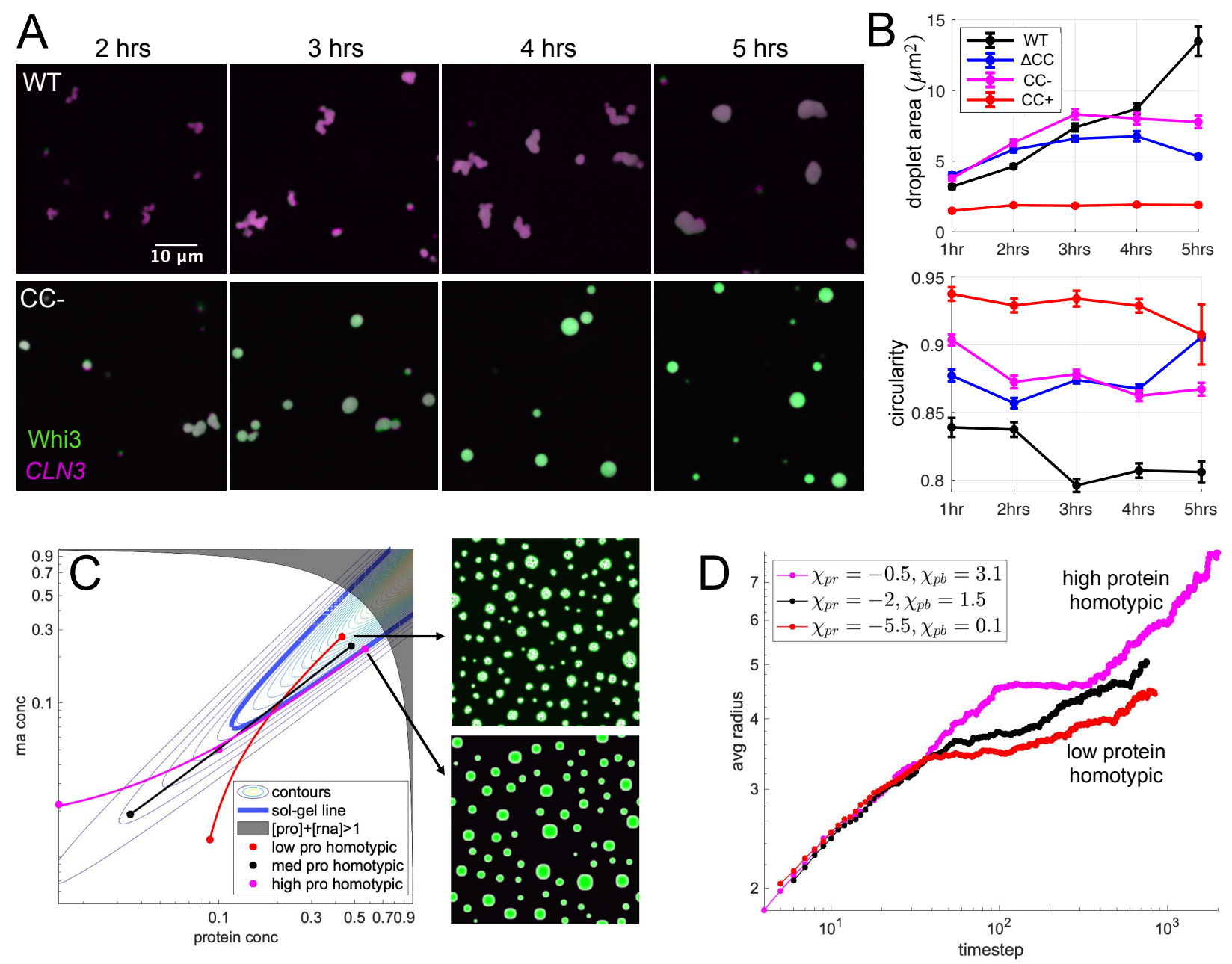

Figure 5: Droplet dynamics are linked to dense phase stoichiometries. (A) Representative images for each time point for WT and Whi3 $\left(\mathrm{CC} \rightarrow \mathrm{CC}^{-}\right)$proteins at bulk concentrations of 500 $\mathrm{nM}$ Whi3 with $5 \mathrm{nM} C L N 3$. (B) Quantification of average droplet area and circularity for 3 replicates of experiments corresponding to panel (A). Error bars are standard error of the mean. (C) For fixed bulk concentrations of protein and RNA, alteration of the protein homotypic interaction energies results in dense phase concentrations that lie at varying depths beyond the solgel line. (D) Simulations with dense phase concentrations further beyond the sol-gel line experience arrested droplet growth at earlier times. 


\section{Discussion}

We have shown that a 21-residue motif, designated as $\mathrm{CC}$, which lies within the Whi3 QRR, controls phase behavior by sequestering the Whi3 protein into dilute phase oligomers. In WT Whi3, the CC motif is likely defined by an equilibrium between helical and non-helical conformations. Mutations that alter the helicity of this motif can be used to tilt the equilibrium toward conformations that are more helical or less helical. Because the CC motif is embedded within the QRR, altering its helical propensity results in trade-offs of order and disorder at the molecular level. Interestingly, these differences at the molecular level have profound effects on the phase behavior of the Whi3-CLN3 system. Interactions among Whi3 molecules with stable helical CC motifs lead to a sequestration of proteins into dilute phase oligomers. This leads to a lowering of protein concentrations in the dense phases of RNP condensates. It also contributes to a faster aging of RNP condensates because the gap between the sol-gel line and dense phase arm of the phase boundary is widened. In contrast, increased disorder within the CC motif and disorder within the remainder of the QRR weakens dilute phase oligomerization and drives Whi3 molecules into RNP condensates. Increased protein concentrations lead to a narrowing of the gap between the sol-gel line and dense phase arm of the phase boundary. This in turn weakens gelation without phase separation and leads to the rapid equilibration via phase separation and gelation that gives rise to network fluids that behave like viscous liquids.

Interestingly, we find that the Whi3 $(\triangle \mathrm{CC})$ construct behaved most like the Whi3 $(\mathrm{CC} \rightarrow$ $\left.\mathrm{CC}^{-}\right)$construct. Specifically, we observe higher protein concentrations in the dense phase for Whi3 $(\triangle \mathrm{CC})$ when compared to WT at similar bulk concentrations of each protein and hence lower bulk volume fractions of Whi3 ( $\triangle \mathrm{CC}$ ) compared to WT. Clearly, the effect of removing the CC is strong enough to compensate for a small length difference that should weaken phase separation of Whi3 $(\triangle \mathrm{CC})$. LASSI simulations suggest that removal of the $\mathrm{CC}$ bead leads to weaker phase separation by 4-bead QRR chains (Fig. S7, SI Appendix). This does not square with the experimental data and is consistent with the ultra-coarse-grained nature of the LASSI model. We hypothesize that there is likely to be weak propagation of the helicity that goes beyond the CC motif and extends into some portion of the N-terminal end of the QRR. Indeed, there is precedent for the propagation of helicity into QRRs as has been shown for different Q-rich systems (68). This would also explain the distinct properties of the Whi3 $(\triangle \mathrm{CC})$ system. Deletion of the CC motif would imply that the QRR seldom samples helical conformations, and phase separation is driven via interactions among disordered regions. For WT, we hypothesize that helicity from the CC propagates into some fraction of the adjacent QRR, tipping the balance toward interactions among helical domains that sequester Whi3 proteins into dilute phase oligomers.

The sequence motifs we have identified within the Whi3 QRR are a fairly common feature of proteins with QRRs. Indeed, other groups have shown that Leu, Pro, and His are especially enriched within and around QRRs, with Leu being typically clustered at N-terminal regions and Pro being prevalent at C-terminal regions $(69,70)$. Here, we observe similar positioning of these residues within the Whi3 QRR (Fig. 1A). In accord with our findings, Kokona and Fairman have shown that formation of stable coiled coils suppresses polyQ driven insolubility via aggregation and the formation of solid-like inclusions by peptides that mimic the exon 1 encoded region of huntingtin (71). Therefore, the interplay of helicity, oligomerization, unfolding, and the driving forces for phase separation might be a general feature of proteins with QRRs.

Relation to other systems with oligomerization domains and IDRs. Many proteins that have been identified as a drivers of phase separation contain both structured oligomerization 
domains and IDRs. Reports to date have concluded that oligomerization promotes phase separation $(2-4,12,37-39,48,72)$. A notable exception to these findings is the prediction of a "magic-number effect" that occurs for rigid domains with sufficiently strong, specific interactions, whereby molecules become trapped in stoichiometric assemblies (73). Similar effects are realizable, irrespective of the strength of inter-domain interactions, if the linkers between the interacting domains are too short (18). In the model that shows the magic number effect, binding partners with an integer multiple of one-to-one binding sites can saturate interactions with each other, favoring the formation of small oligomers in the dilute phase that weakens phase separation. Increasing rigidity of participating polymer species enhances this effect. The experiments we summarize here show the phenomenon of dilute phase oligomers sequestering the Whi3 protein out of the dense phase, although it is unclear whether this is due to a magic-number effect. It is possible that the CC motif may prefer a specific oligomerization state, which on its own could conceivably result in stable oligomers with saturated binding sites. However, Whi3 also mediates homotypic interactions through the rest of its QRR that presumably has no fixed valence. It also participates in heterotypic interactions with RNA via its RRM and the QRR has the potential for engaging complementary IDRs of other proteins in cells (49). These additional interactions contribute complexity to the system that complicates interpretation in the context of a magic number effect, although the phenomenological similarity between the effects of increasing the CC stability (rigidity) and interaction energy and the effects seen in the theory are intriguing.

Cryptic motifs as regulators of phase separation. Negative regulation of phase separation by motifs that undergo linked conformational and oligomerization changes in the dilute phase may be a general mechanism by which IDRs regulate phase transitions. Here, we have identified macroscopic consequences of sequence-level changes in one of the components of a multi-component system. Resulting condensates had altered dense phase concentrations and distinct material properties. Control of viscoelasticity can result in tuned pore size, condensate size distributions, and lifetimes of condensates, all of which may need to be specifically calibrated as part of normal cell functioning.

Interestingly, we also observe that dilute phase oligomers contribute, via linked equilibria, to changes within the dense phase. Accordingly, it follows that the oligomer distribution in the dilute phase may be simultaneously tuned as part of cell functioning. Outside of normal physiology, the presence of cryptic structural motifs being embedded within IDRs may provide a rationale for benign-looking disease-related point mutations, as was shown for TDP-43 (48). If such mutations perturb regulatory structural motifs and their associations, then drastic shifts in phase behavior and material states are possible even with very few residue changes. Importantly, the effects of the CC motif that we have uncovered in this study are distinct from those in TDP43, highlighting the need for context-dependent studies of embedded structured domains. Identification of such motifs within phase separating systems will be an essential task for understanding regulatory control of biomolecular condensates.

\section{Materials and Methods}

Details of the simulations and all of the experiments are described in the SI Appendix.

CAMPARI simulations: All-atom simulations were performed using version 3.0 of CAMPARI modeling suite (http://campari.sourceforge.net). Forcefield parameters were taken from the abs_opls_3.2.prm set. Simulations were performed on the Longleaf computer cluster at 
UNC-Chapel Hill. Default parameters were used for the forcefield and Monte Carlo move sets (56). Details of the simulation setup and analysis modules are described in the SI Appendix.

UV-Circular Dichroism: Peptides were purchased in pure form from GenScript with Nterminal acetylation and C-terminal amidation. All peptide sequences included an N-terminal tryptophan to enable concentration measurements, followed by a glycine, and then the sequence of interest. CD measurements were carried out using a Jasco 810 spectropolarimeter scanning from $260 \mathrm{~nm}$ to $190 \mathrm{~nm}$, with a data pitch of $1 \mathrm{~nm}$ and a bandwidth of $1 \mathrm{~nm}$. Four to six accumulations were averaged for each spectrum with a scanning speed of $50 \mathrm{~nm} / \mathrm{min}$ and a two second response time. Additional details regarding the CD measurements are described in the SI Appendix.

Assembly and imaging of Whi3-CLN3 condensates in vitro: Whi3 protein (WT, $\mathrm{CC}+$, $\mathrm{CC}-, \Delta \mathrm{CC}$ ) and $C L N 3 \mathrm{mRNA}$ were stored in $200 \mu \mathrm{L}$ and $10 \mu \mathrm{L}$ aliquots respectively at $-80^{\circ} \mathrm{C}$ until just before imaging experiments. Aliquots were thawed on ice and protein samples were spun down at 13,200 rpm for 5 minutes to remove any aggregates. The concentration of protein was then determined by measuring absorbance at $280 \mathrm{~nm}$. Whi3 proteins and CLN3 mRNA were diluted into buffer containing $50 \mathrm{mM}$ HEPES at $\mathrm{pH} 7.4$ and $150 \mathrm{mM} \mathrm{KCl}$ to desired concentrations of protein and mRNA. Whi3 proteins were prepared at three concentrations, 250nM, 500nM, and $1 \mu \mathrm{M}$, and $C L N 3 \mathrm{mRNA}$ was at a concentration of $5 \mathrm{nM}$ for every condition. $200 \mu \mathrm{L}$ of protein and mRNA solutions were loaded into glass-bottom imaging chambers (Grace Bio-Labs) that were pre-coated with $30 \mathrm{mg} / \mathrm{mL}$ BSA (Sigma) for 30 mins to prevent protein adsorption to the well surfaces and washed thoroughly with experiment buffer prior to addition of protein and mRNA solutions. Whi3 was added first to imaging wells, followed by $C L N 3$, and the final solution was mixed by pipetting without introducing bubbles. Imaging chambers were immediately transitioned to a temperature-controlled microscope stage (Tokai Hit from Incubation System for Microscopes) set to $25 \mathrm{C}$. Every hour, starting one hour after sample preparation, confocal z-stacks of imaging wells were acquired. Three z-stacks within each well were taken at each time point at random locations. Fluorescence images in two channels were acquired using $488 \mathrm{~nm}$ and $561 \mathrm{~nm}$ lasers to visualize Atto488-labeled Whi3 and Cy3-labeled CLN3 respectively. In Whi3-CLN3 experiments, different Whi3 proteins (WT, $\mathrm{CC}-, \mathrm{CC}+, \Delta \mathrm{CC}$ ) did not have fixed labeling ratios. For these experiments, a laser power and exposure time was optimized for each protein based on the fluorescence of free Whi3 imaged in wells at multiple concentrations in $150 \mathrm{mM} \mathrm{KCl}$ where Whi3 alone does not phase separate. These conditions were used to create calibration curves to determine concentrations of protein based on fluorescence intensity. Since the same CLN3 stock was used for all experiments, the labeling ratio is constant among all experiments and the fluorescence intensities in the CLN3 channel can be directly compared among experiments; hence, we did not compute calibration curves for $C L N 3$ to determine concentrations. Experiments were replicated three times.

LASSI simulations: Simulations were performed using LASSI and run on the Longleaf computing cluster at UNC-Chapel Hill. Each simulation was run independently on a single compute node with 4GB RAM. Interaction energies that were used for each of the three systems studied (WT, CC+, CC-), are shown in Table S2 SI Appendix.

ACKNOWLEDGMENTS. This work was supported by grants from the US National Institutes of Health (5R01NS056114 and R01NS089932 to RVP, R01BM081506 to ASG, F32GM133123-01A1 to BMS, F32-GM136055 to WTS, and T32-GM8570-25 to IS), the Air Force Office of Scientific Research (FA9550-20-1-0241 to ASG and RVP), the HHMI faculty scholars 
(to ASG). We are grateful to Furqan Dar, Alex Holehouse, and Kiersten Ruff for helpful discussions and assistance with LASSI as well as CAMPARI simulations.

\section{References}

1. S. F. Banani, H. O. Lee, A. A. Hyman, M. K. Rosen, Biomolecular condensates: organizers of cellular biochemistry. Nature Reviews Molecular Cell Biology 18, 285-298 (2017).

2. P. Yang et al., G3BP1 Is a Tunable Switch that Triggers Phase Separation to Assemble Stress Granules. Cell 181, 325-345 e328 (2020).

3. D. W. Sanders et al., Competing Protein-RNA Interaction Networks Control Multiphase Intracellular Organization. Cell 181, 306-324.e328 (2020).

4. J. Guillen-Boixet et al., RNA-Induced Conformational Switching and Clustering of G3BP Drive Stress Granule Assembly by Condensation. Cell 181, 346-361 e317 (2020).

5. C. J. Decker, R. Parker, P-Bodies and Stress Granules: Possible Roles in the Control of Translation and mRNA Degradation. Cold Spring Harbor Perspectives in Biology 4 (2012).

6. W. Xing, D. Muhlrad, R. Parker, M. K. Rosen, A quantitative inventory of yeast P body proteins reveals principles of composition and specificity. eLife $\mathbf{9}$, e56525 (2020).

7. J. A. Greig et al., Arginine-Enriched Mixed-Charge Domains Provide Cohesion for Nuclear Speckle Condensation. Molecular Cell 77, 1237-1250.e1234 (2020).

8. C. P. Brangwynne, T. J. Mitchison, A. A. Hyman, Active liquid-like behavior of nucleoli determines their size and shape in Xenopus laevis oocytes. Proceedings of the National Academy of Sciences USA 108, 4334-4339 (2011).

9. E. M. Langdon et al., mRNA structure determines specificity of a polyQ-driven phase separation. Science 360, 922 (2018).

10. S. Boeynaems et al., Spontaneous driving forces give rise to protein-RNA condensates with coexisting phases and complex material properties. Proceedings of the National Academy of Sciences USA 116, 7889-7898 (2019).

11. S. Maharana et al., RNA buffers the phase separation behavior of prion-like RNA binding proteins. Science 360, 918-921 (2018).

12. J. A. Riback et al., Composition-dependent thermodynamics of intracellular phase separation. Nature 581, 209-214 (2020).

13. I. Alshareedah, M. M. Moosa, M. Raju, D. A. Potoyan, P. R. Banerjee, Phase transition of RNA-protein complexes into ordered hollow condensates. Proceedings of the National Academy of Sciences 117, 15650 (2020). 
14. J.-M. Choi, F. Dar, R. V. Pappu, LASSI: A lattice model for simulating phase transitions of multivalent proteins. PLoS computational biology 15 (2019).

15. J.-M. Choi, A. S. Holehouse, R. V. Pappu, Physical Principles Underlying the Complex Biology of Intracellular Phase Transitions. Annual Review of Biophysics 49, 107-133 (2020).

16. J.-M. Choi, A. A. Hyman, R. V. Pappu, Generalized models for bond percolation transitions of associative polymers. Physical Review E 102, 042403 (2020).

17. J. Wang et al., A Molecular Grammar Governing the Driving Forces for Phase Separation of Prion-like RNA Binding Proteins. Cell 174, 688-699. e616 (2018).

18. T. S. Harmon, A. S. Holehouse, M. K. Rosen, R. V. Pappu, Intrinsically disordered linkers determine the interplay between phase separation and gelation in multivalent proteins. eLife 6, e30294 (2017).

19. T. S. Harmon, A. S. Holehouse, R. V. Pappu, Differential solvation of intrinsically disordered linkers drives the formation of spatially organized droplets in ternary systems of linear multivalent proteins. New Journal of Physics 20, 045002 (2018).

20. M. C. Cohan, R. V. Pappu, Making the Case for Disordered Proteins and Biomolecular Condensates in Bacteria. Trends in Biochemical Sciences 45, 668-680 (2020).

21. P. Li et al., Phase transitions in the assembly of multivalent signalling proteins. Nature 483, 336-340 (2012).

22. Timothy J. Nott et al., Phase Transition of a Disordered Nuage Protein Generates Environmentally Responsive Membraneless Organelles. Molecular Cell 57, 936-947 (2015).

23. R. M. Vernon et al., Pi-Pi contacts are an overlooked protein feature relevant to phase separation. eLife 7, e31486 (2018).

24. J. P. Brady et al., Structural and hydrodynamic properties of an intrinsically disordered region of a germ cell-specific protein on phase separation. Proc Natl Acad Sci U S A 114, E8194-E8203 (2017).

25. T. H. Kim et al., Phospho-dependent phase separation of FMRP and CAPRIN1 recapitulates regulation of translation and deadenylation. Science 365, 825 (2019).

26. E. W. Martin et al., Valence and patterning of aromatic residues determine the phase behavior of prion-like domains. Science 367, 694-699 (2020).

27. T. Kaur et al., Sequence-encoded and composition-dependent protein-RNA interactions control multiphasic condensate morphologies. Nature Communications 12, 872 (2021). 
28. C. Roden, A. S. Gladfelter, RNA contributions to the form and function of biomolecular condensates. Nature Reviews Molecular Cell Biology 22, 183-195 (2021).

29. S. F. Banani et al., Compositional Control of Phase-Separated Cellular Bodies. Cell 166, 651-663 (2016).

30. X. Su et al., Phase separation of signaling molecules promotes $\mathrm{T}$ cell receptor signal transduction. Science 352, 595-599 (2016).

31. P. St George-Hyslop et al., The physiological and pathological biophysics of phase separation and gelation of RNA binding proteins in amyotrophic lateral sclerosis and fronto-temporal lobar degeneration. Brain Research 1693, 11-23 (2018).

32. C. P. Brangwynne, P. Tompa, R. V. Pappu, Polymer physics of intracellular phase transitions. Nature Physics 11, 899-904 (2015).

33. H. Zhang et al., RNA controls PolyQ protein phase transitions. Molecular Cell 60, 220230 (2015).

34. C. Iserman et al., Genomic RNA Elements Drive Phase Separation of the SARS-CoV-2 Nucleocapsid. Molecular Cell 80, 1078-1091.e1076 (2020).

35. J. Cubuk et al., The SARS-CoV-2 nucleocapsid protein is dynamic, disordered, and phase separates with RNA. Nature Communications 12, 1936 (2021).

36. K. B. Shpargel, J. K. Ospina, K. E. Tucker, A. G. Matera, M. D. Hebert, Control of Cajal body number is mediated by the coilin C-terminus. Journal of Cell Science 116, 303 (2003).

37. S. K. Powers et al., Nucleo-cytoplasmic Partitioning of ARF Proteins Controls Auxin Responses in Arabidopsis thaliana. Molecular Cell 76, 177-190.e175 (2019).

38. Y. Shin et al., Spatiotemporal Control of Intracellular Phase Transitions Using LightActivated optoDroplets. Cell 168, 159-171.e114 (2017).

39. D. Bracha et al., Mapping Local and Global Liquid Phase Behavior in Living Cells Using Photo-Oligomerizable Seeds. Cell 175, 1467-1480.e1413 (2018).

40. S. Roberts et al., Injectable tissue integrating networks from recombinant polypeptides with tunable order. Nature Materials 17, 1154-1163 (2018).

41. A. Toto et al., Binding induced folding: Lessons from the kinetics of interaction between NTAIL and XD. Archives of Biochemistry and Biophysics 671, 255-261 (2019).

42. K. Bugge et al., $\alpha \alpha-H u b$ domains and intrinsically disordered proteins: A decisive combo. Journal of Biological Chemistry 296, 100226 (2021). 
43. H. J. Dyson, P. E. Wright, Role of Intrinsic Protein Disorder in the Function and Interactions of the Transcriptional Coactivators CREB-binding Protein (CBP) and p300*. Journal of Biological Chemistry 291, 6714-6722 (2016).

44. S. L. Shammas, M. D. Crabtree, L. Dahal, B. I. M. Wicky, J. Clarke, Insights into Coupled Folding and Binding Mechanisms from Kinetic Studies*. Journal of Biological Chemistry 291, 6689-6695 (2016).

45. R. Vancraenenbroeck, Y. S. Harel, W. Zheng, H. Hofmann, Polymer effects modulate binding affinities in disordered proteins. Proceedings of the National Academy of Sciences 116, 19506 (2019).

46. T. R. Sosnick, S. Jackson, R. R. Wilk, S. W. Englander, W. F. DeGrado, The role of helix formation in the folding of a fully $\alpha$-helical coiled coil. Proteins: Structure, Function, and Bioinformatics 24, 427-432 (1996).

47. E. Dürr, H. R. Bosshard, Folding of a three-stranded coiled coil. Protein Science 9, 14101415 (2000).

48. A. E. Conicella et al., TDP-43 $\alpha$-helical structure tunes liquid-liquid phase separation and function. Proceedings of the National Academy of Sciences 117, 5883 (2020).

49. C. Lee, P. Occhipinti, A. S. Gladfelter, PolyQ-dependent RNA-protein assemblies control symmetry breaking. Journal of Cell Biology 208, 533-544 (2015).

50. A. N. Lupas, M. Gruber, "The Structure of $\alpha$-Helical Coiled Coils" in Advances in Protein Chemistry. (Academic Press, 2005), vol. 70, pp. 37-38.

51. M. Gruber, J. Söding, A. N. Lupas, Comparative analysis of coiled-coil prediction methods. Journal of Structural Biology 155, 140-145 (2006).

52. Y. Cheng et al., Mining $\alpha$-Helix-Forming Molecular Recognition Features with Cross Species Sequence Alignments. Biochemistry 46, 13468-13477 (2007).

53. C. J. Oldfield et al., Coupled Folding and Binding with $\alpha$-Helix-Forming Molecular Recognition Elements. Biochemistry 44, 12454-12470 (2005).

54. A. Vitalis, R. V. Pappu, ABSINTH: A new continuum solvation model for simulations of polypeptides in aqueous solutions. Journal of Computational Chemistry 30, 673-699 (2009).

55. T. S. Harmon et al., GADIS: Algorithm for designing sequences to achieve target secondary structure profiles of intrinsically disordered proteins. Protein Engineering, Design and Selection 29, 339-346 (2016).

56. R. K. Das, S. L. Crick, R. V. Pappu, N-Terminal Segments Modulate the $\alpha$-Helical Propensities of the Intrinsically Disordered Basic Regions of bZIP Proteins. Journal of molecular biology 416, 287-299 (2012). 
57. M. A. Kelly et al., Host-Guest Study of Left-Handed Polyproline II Helix Formation. Biochemistry 40, 14376-14383 (2001).

58. J. M. Scholtz et al., Calorimetric determination of the enthalpy change for the alpha-helix to coil transition of an alanine peptide in water. Proceedings of the National Academy of Sciences 88, 2854 (1991).

59. D. W. Urry, T. H. Ji, Distortions in circular dichroism patterns of particulate (or membranous) systems. Archives of Biochemistry and Biophysics 128, 802-807 (1968).

60. D. W. Urry, J. Krivacic, Differential Scatter of Left and Right Circularlay Polarized Light by Optically Active Particulate Systems. Proceedings of the National Academy of Sciences 65, 845 (1970).

61. L. N. M. Duyens, The flattering of the absorption spectrum of suspensions, as compared to that of solutions. Biochimica et Biophysica Acta 19, 1-12 (1956).

62. A. Z. Akcasu, I. C. Sanchez, Foundations and Extensions of the Cahn-Hilliard-Cook Theory. Molecular Crystals and Liquid Crystals Incorporating Nonlinear Optics 180, 147153 (1990).

63. X. Zeng, A. S. Holehouse, A. Chilkoti, T. Mittag, R. V. Pappu, Connecting Coil-to-Globule Transitions to Full Phase Diagrams for Intrinsically Disordered Proteins. Biophysical Journal 119, 402-418 (2020).

64. F. Sciortino, R. Bansil, H. E. Stanley, P. Alstrom, Interference of Phase-Separation and Gelation - a Zeroth-Order Kinetic-Model. Physical Review E 47, 4615-4618 (1993).

65. E. Zaccarelli, P. J. Lu, F. Ciulla, D. A. Weitz, F. Sciortino, Gelation as arrested phase separation in short-ranged attractive colloid-polymer mixtures. J Phys-Condens Mat 20 (2008).

66. K. Rhine et al., ALS/FTLD-Linked Mutations in FUS Glycine Residues Cause Accelerated Gelation and Reduced Interactions with Wild-Type FUS. Molecular Cell 80, 666-681.e668 (2020).

67. I. M. Lifshitz, V. V. Slyozov, The kinetics of precipitation from supersaturated solid solutions. Journal of Physics and Chemistry of Solids 19, 35-50 (1961).

68. A. Escobedo et al., Side chain to main chain hydrogen bonds stabilize a polyglutamine helix in a transcription factor. Nature Communications 10, 2034 (2019).

69. M. Ramazzotti, E. Monsellier, C. Kamoun, D. Degl'Innocenti, R. Melki, Polyglutamine Repeats Are Associated to Specific Sequence Biases That Are Conserved among Eukaryotes. PLOS ONE 7, e30824 (2012). 
70. A. Urbanek et al., Flanking Regions Determine the Structure of the Poly-Glutamine in Huntingtin through Mechanisms Common among Glutamine-Rich Human Proteins. Structure 28, 733-746.e735 (2020).

71. B. Kokona, Z. P. Rosenthal, R. Fairman, Role of the Coiled-Coil Structural Motif in Polyglutamine Aggregation. Biochemistry 53, 6738-6746 (2014).

72. M. C. Ferrolino, D. M. Mitrea, J. R. Michael, R. W. Kriwacki, Compositional adaptability in NPM1-SURF6 scaffolding networks enabled by dynamic switching of phase separation mechanisms. Nature Communications 9, 5064 (2018).

73. B. Xu et al., Rigidity enhances a magic-number effect in polymer phase separation. Nature Communications 11, 1561 (2020). 